\title{
Analysis of two putative regulatory elements of Myostatin gene in $\mathrm{C}_{2} \mathrm{C}_{12}$ myoblasts
}

\author{
Lúcia E. Alvares (PQ), Marina A. Fontoura (IC), Carolina S. Mantovani (PG). \\ Laboratory of Developmental Biology, Department of Biochemistry and Tissue Biology. Institute of \\ Biology - State University of Campinas
}

\begin{abstract}
This research aims to verify the function of defined ECRs found in Myostatin gene locus during myogenesis.
\end{abstract}

Key words: Myostatin, myoblasts, evolutionary conserved regions.

\section{Introduction}

Muscle development is negatively regulated by the protein Myostatin (Mstn), a member of TGF- $\beta$ superfamily of transforming and growth factors ${ }^{\uparrow}$. Our research team performed comparative genomic analysis in order to locate evolutionary conserved regions (ECRs) adjacent to the locus of the Mstn gene in human (Homo sapiens), mouse (Mus musculus) and chicken (Gallus gallus) ${ }^{2}$. We found six ECRs that may represent regulatory elements of the Mstn gene once they have been maintained across amniote genome evolution. Moreover, two of those ECRs have conserved binding sites for myogenic factors, suggesting that they could be related to skeletal muscle development.

In this research, we tested the functional potential of those ECRs in $\mathrm{C}_{2} \mathrm{C}_{12}$ myoblasts by transfecting these cells with plasmids composed of each ECR controlling the expression of GFP reporter gene. We analysed our data using flow cytometry and quantitative PCR.

\section{Results and Discussion}

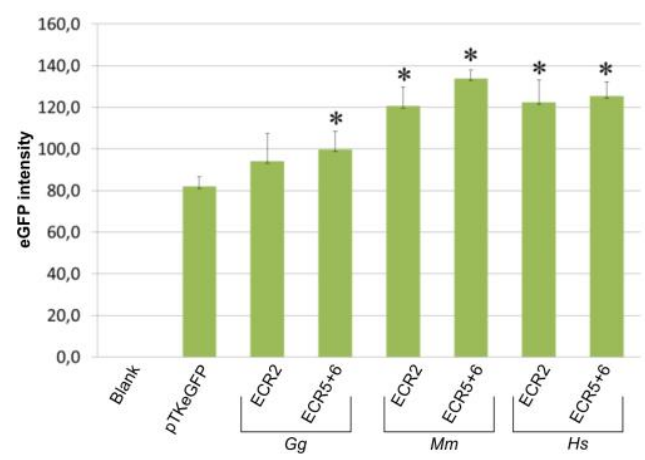

Figure 1. Fluorescence intensity of each experimental group of transfected $\mathrm{C}_{2} \mathrm{C}_{12}$ cells in $60-70 \%$ of confluence. ${ }^{*} \mathrm{p} \leq 0,05$. Average percent transfection: $40 \%$.

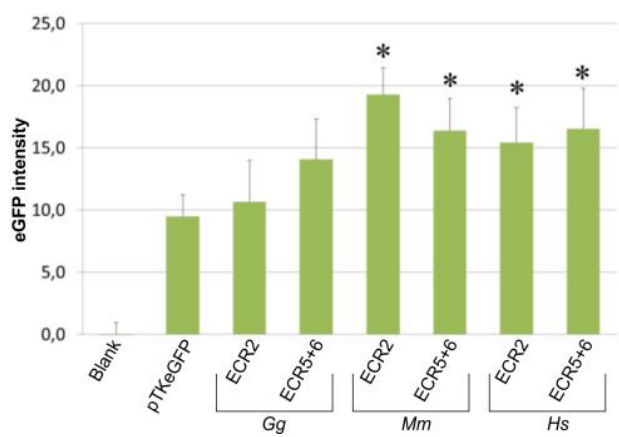

Figure 2. Fluorescence intensity of each experimental group of transfected $\mathrm{C}_{2} \mathrm{C}_{12}$ cells in $>90 \%$ of confluence. ${ }^{*} \mathrm{p} \leq 0,05$. Average percent transfection: $4 \%$.

eGFP expression levels were increased in constructs with ECR2 and ECR5+6 of mouse and human, whereas chicken's ECR2 resulted in no significant changes in the expression of the reporter gene. Chicken's ECR5+6 produced a significant result only in the first experiment in which cells presented a lower level of differentiation, which points out the need to study the specific roles of these elements during proliferation and differentiation of myoblasts to better understand the functionality of these ECRs.

\section{Conclusions}

The functional analysis of human and mouse's ECRs confirmed their potential as regulatory elements in the myogenic context since they showed the ability to modulate the expression of the GFP reporter. Further analyses are necessary to confirm their role specifically in the regulation of the Mstn gene expression.

\section{Acknowledgement}

I thank the National Council of Scientific and Technological Development (CNPq) for granting the Scientific Initiation scholarship.

${ }^{1}$ McPherron, A.C.; Lawler, A.M.; Lee, S.J. Nature. 1997, 387, 6628 ${ }^{2}$ Grade, C.V.; Salerno, M.S.; Schubert, F.R.; Dietrich, S.; Alvares, L.E. Dev. Genes Evo. 2009, 219, 497 